\title{
Hypovitaminosis D and the endocrine phenotype of COVID-19
}

\author{
Andrea Giustina $\mathbb{B}^{1}$
}

Received: 4 February 2021 / Accepted: 24 February 2021 / Published online: 18 March 2021

(c) The Author(s), under exclusive licence to Springer Science+Business Media, LLC, part of Springer Nature 2021

\begin{abstract}
Background Vitamin D and its deficiency have recently been suspected to be involved in increased susceptibility and negative outcomes of COVID-19. This assumption was based on the well known immunomodulatory actions of vitamin D and on the consistent finding of low levels of 25 hydroxyvitamin D (25OHD) in hospitalized patients with COVID-19. Moreover, several studies reported a correlation between 25OHD levels and different clinical outcomes of the disease.

Aim Aim of the current review was to approach the topic of vitamin D and COVID-19 from a different perspective summarizing the data which led to the evidence of the existence of an endocrine phenotype of COVID-19.

Conclusions This review analyzed in the light of the current knowledge the possibility that several endocrine manifestations of COVID-19 could be holistically interpreted in the context of an inadequate vitamin D status.
\end{abstract}

Keywords Vitamin D $\cdot$ Hypocalcemia $\cdot$ Vertebral fractures $\cdot$ Diabetes mellitus $\cdot$ Obesity $\cdot$ COVID-19

\section{Introduction}

COVID-19 has been immediately defined as an often severe respiratory infection caused by severe acute respiratory syndrome coronavirus 2 (SARS-CoV-2) but with time important extrapulmonary involvement highly contributing to the lethality of the resulting syndrome has been described [1]. Despite the origin and first outbreak of the infection being apparently in China, Mediterranean Countries such as Italy and Spain have been rapidly involved in the pandemic and are currently paying the highest death toll to COVID-19 infection in the whole world. Specifically, in Italy deaths are reaching today the impressive number of 100.000 , about $3 \%$ of the 3 millions of known affected citizens (covid19@gimbe.org, March 2021). The most relevant extrapulmonary conditions which impact on the severity of the disease include mainly thrombotic complications leading to myocardial infarction and dysfunction with arrhythmias, but also renal, gastrointestinal, liver, central nervous system, eye, and skin complications have been well described so far [1]. Pathophysiological basis for these pleiotropic manifestations

Andrea Giustina

giustina.andrea@hsr.it

1 Institute of Endocrine and Metabolic Sciences, San Raffaele, VitaSalute University and IRCCS Hospital, Milan, Italy of COVID-19 is the expression of angiotensin-converting enzyme 2 (ACE2), the receptor responsible for the cellular entry of SARS-CoV-2, at the level of many tissues besides the lung mediating the multiple damages caused by this viral infection [2]. Additionally, endothelial damages at endothelial and vascular levels as well as deranged immune response are also key in determining the multifacet manifestations of COVID-19 [3].

In this complex and evolving scenario a major role for endocrine diseases has progressively emerged. In fact, with accumulating experience on the clinical presentation of the disease it shaped up what we can call an "endocrine phenotype" of COVID-19 with many endocrine organs, tissues, and molecules being at risk of direct or indirect alterations and, in turn, negatively impacting COVID-19 $[4,5]$.

Aim of this article will be to shortly review the major endocrine comorbidities as well as some other related factors associated with COVID-19 with an emphasis on hypovitaminosis $\mathrm{D}[6]$ also in the light of the latter being the possible link between all of them and therefore representing phil rouge of the endocrine phenotype of COVID-19.

\section{Methods}

For this narrative review and critical analysis of available evidence, literature searches were performed by author to identify new data in English language papers published 
between January and December 2020, and indexed in PubMed. Search terms included "COVID-19" and terms associated with each topic, including "endocrine glands", "gender", "age", "diabetes", "obesity", "body composition", "vitamin D", "hypocalcemia", "vertebral fractures", "hypovitaminosis D".

\section{Review of the evidences}

\section{Endocrine comorbidities and other conditions associated with COVID-19 severity}

\section{Aging and sex}

Aging Besides sex, age appeared to be a key factor in viral lethality. In fact, mortality data from Italy clearly indicated that most deaths occurred after the age of 70 years old, with about half of them occurring in the age decade from 80 to 89 years [7]. In fact, it was initially supposed that the high lethality of COVID-19 in Italy could have been linked to the high number of elderly people leading to the unlikely assumption that Italians were dying with COVID-19 rather than for COVID-19 [8].

Sex From the beginning of the COVID-19 pandemic it appeared clear how the outcome of the disease was largely sex dependent. In fact, men and women appeared to be equally affected, but men were at higher risk of a more severe disease as compared to women with a similar pattern in different countries. In a highly impacted country such as Italy male sex was associated with a more aggressive disease and increased mortality risk [7].

\section{Diabetes mellitus and diabetic retinopathy}

Diabetes mellitus From the first days of the COVID outbreak it was clear that one of the most frequent comorbidities of hospitalized patients was diabetes mellitus. However, progressively, it became apparent that between diabetes and COVID-19 there could be a bidirectional relationship [9].

According to different case series diabetes is seen in patients with COVID-19 with a prevalence ranging from 7 to $30 \%$. In term of outcomes, persons with diabetes with SARSCoV-2 infection are at higher risk of hospitalization, severe pulmonary involvement, and mortality with respect to nondiabetic subjects [10]. Interestingly, in a retrospective, nationwide analysis of registries of 157,291 patients with adult community acquired pneumonia in Portugal, from 2009 to $2012,23.7$ to $28.1 \%$ presented diabetes as a comorbidity, but hospital mortality was only slightly higher in diabetic $(15.2 \%)$ vs nondiabetic (13.5\%) patients [11].
Poor glyco-metabolic control at admission and during hospitalization has negative impact on outcomes and levels of disease activity markers as interleukin (IL)-6 and D-dimer are higher in hyperglycemic vs euglycemic patients [12]. Pathophysiologically, chronically elevated blood glucose may negatively impact both humoral and innate immunity as well as associate with low-grade inflammation, which, in turn, may predispose to an excessive inflammatory reaction leading to acute respiratory distress syndrome. Hyperglycemia may also negatively impact on the lung inducing several structural alterations leading to increased alveolo-capillary permeability and collapse of alveolar epithelium; consequently, diabetes may worsen respiratory damages caused by COVID-19 [5].

Interestingly, recent evidence suggested that pancreatic $\beta$-cells, which express the ACE2 receptor, may be directly affected by SARS-CoV-2 causing a more severe hyperglycemia with frequent episodes of ketoacidosis and hyperosmolarity in need of high-dose insulin treatment. Moreover, newly onset diabetes in previously euglycemic subjects was consistently reported in patients with COVID19 [9] pointing toward a potential diabetogenic effect of SARS-CoV-2 [12]. The transitory or permanent nature of hyperglycemia in COVID-19 as well as the real incidence and type of this newly onset diabetes are all currently matters of investigation and a global registry is collecting those cases worldwide in the frame of an international collaborative project called CoviDIAB [9].

Diabetic retinopathy Recently, the CORONADO study demonstrated that a composite microvascular diabetic damage index, which included severe retinopathy and/or diabetic food and/or nephropathy predicted early mortality in COVID-19 patients [13].

Interestingly, a recent monocentric cross-sectional study on 187 hospitalized patients with poorly controlled diabetes and COVID-19 found that retinopathy, as well as obesity, was positively associated with intubation. Moreover, at multivariate analysis retinopathy was independently associated with an increased risk for intubation with a statistically significant odd ratio slightly lower than 6 . Due to the nature of the study, causal link or underlying mechanism of the association was not established. However, authors speculated that diabetic microangiopathy could have been a surrogate marker of a preexisting endothelial dysfunction/damage which may represent a risk factor for an increased severity of respiratory dysfunction in COVID-19 [14].

\section{Obesity and body composition}

Obesity increases risk for hospitalization, ICU admission, mechanical ventilation, and mortality in COVID-19 patients. 
Moreover, excess in visceral adipose tissue may be a negative prognostic factor in people with COVID-19 [5]. Interestingly, acute sarcopenia [15] appears to often characterize survivors of COVID-19 [16].

In fact, particularly severe obesity was consistently reported as one of the major predictors of the need of hospitalization in COVID-19 due to the severity of pneumonia. Severe obesity was also associated with requirement of invasive ventilation and death in ICU $[5,17]$.

A recent meta-analysis, which included more than 45,000 patients [18], showed both at univariate and multivariate analyses that obesity was related to a significantly increased (approximately doubled) risk of severe COVID-19, with similar impact on all evaluated clinical outcomes (hospitalization, admission in ICU, need for mechanical ventilation, and death). Highest obesity-related risk at multivariate analysis was found for mechanical ventilation (odd ratio 2.63). Interestingly, visceral adiposity was found to be increased in severe vs non-severe COVID-19 patients and significantly associated with all evaluated clinical outcomes (need for hospitalization, mechanical ventilation, and ICU admission).

Pathophysiologically, the high impact of obesity on prognosis in COVID-19 patients is likely related to the deleterious effects of obesity on pulmonary function. In fact, obesity can negatively impact on several parameters of lung function such as expiratory reserve volume, functional capacity, and pulmonary compliance. Severe obesity is also associated with sleep apnea syndrome, which, in turn, is associated with increased cardiovascular risk [19]. Moreover, increased abdominal fat may impair diaphragmatic and respiratory function [20]. Obesity per se may also increase the risk of comorbidities already known to contribute to COVID-19 severity such as diabetes mellitus and atherothrombosis [21].

Interestingly, obesity is also known to be a state of lowgrade inflammation linked to adipocyte hypoxia and impaired function and self-maintaining due to a recruitment of immune cells and macrophages related to baseline excessive secretion of pro-inflammatory cytokines such as tumor necrosis factor $\alpha$, IL-1 $\beta$, and IL-6 [22], which are, in turn, involved in the cytokine storm typical of COVID-19 and likely overproduced in obese vs lean subjects [5, 23].

\section{Vertebral fractures (VFs)}

Recently, we performed a study in order to assess presence and clinical impact of VFs on lateral chest X-rays in 114 COVID-19 patients. Interestingly, we found that VFs were highly prevalent and one of the most frequent comorbidities in our study population, as up to $35 \%$ of patients presented VF with previous diagnosis of osteoporosis in very few of them [24]. In particular, VFs demonstrated to be a strongly impacting prognostic element since overall mortality rate was found to be doubled in patients with vs without VFs and significantly higher in patients with severe VFs as compared to those with mild and moderate VFs.

Briefly, thoracic VFs [25] at hospital admission were detected in 41 of 114 patients (36\%) and were prevalently mild but in $41 \%$ of the cases moderate or severe [26]. Age was significantly associated with VFs in multivariate analysis. Patients with VFs required more frequently hospitalization and noninvasive mechanical ventilation vs patients without VFs. Mortality was doubled in the VFs+ vs VFsgroup (22 vs $10 \%$ ) and statistically higher in patients with severe VFs compared to those with moderate and mild VFs [24]. No sex differences in the VF group were observed.

Pathophysiologically, we hypothesized that the high prevalence of VFs in COVID-19 could have been due to negative VFs impact on respiratory function since prevalent VFs were previously associated with impaired pulmonary (restrictive) function and increased risk of pneumonia in patients without previous lung diseases. It can be thought that VFs and their severity could be an objective clinical marker of frailty and worse outcome in COVID-19 [24].

\section{Hypocalcemia}

Currently, hypocalcemia has emerged as one major biochemical finding in COVID-19. However, all initiated with the report of a single patient with COVID-19 presenting with severe hypocalcemia with possible latent hypoparathyroidism due to previous total thyroidectomy [27]. Subsequently, in a large monocentric study on more than 500 patients with COVID-19 hypocalcemia was reported in three quarters of them [28]. In the same series, hypocalcemia also demonstrated a relevant clinical impact since calcium levels at initial evaluation were lower in finally hospitalized vs nonhospitalized patients, and hypocalcemia was found to be an independent risk factor for hospitalization [28]. Moreover, it is interesting to note the strong association between high LDH and PCR levels with low calcium levels [28]. A subsequent study reported double rate of hypocalcemia with lower calcium levels in clinically matched 20 hospitalized patients for COVID-19 vs 20 nonCOVID-19 patients with acute respiratory diseases suggesting that hypocalcemia may be a distinctive feature of COVID-19 [29].

These findings were substantially confirmed in many following studies which demonstrated: (a) lower calcium levels in patients tested positive to SARS-CoV-2 RT-qPCR with respect to negative patients; (b) negative association between inflammatory parameters, as CRP and LDH levels, with calcium levels; (c) hypocalcemia as an independent risk factor for worse clinical outcome, including not only hospitalization as shown in our cohort but also ICU admission and mortality [30-32]. 
Pathophysiologically, calcium was already well known to play a crucial role in the mechanism of action of enveloped viruses such as SARS-CoV, MERS-CoV, and Ebolavirus, directly interacting with their fusion peptides and favoring their replication [33-35]. Moreover, hypocalcemia was frequently reported in hospitalized SARS-CoV and Ebolavirus patients [36, 37].

\section{Hypovitaminosis D and COVID-19}

Vitamin D (VD) is a steroid hormone produced by the skin when exposed to sunlight as cholecalciferol while nutritional intake does not contribute more than $20 \%$ of daily VD requirement if food is not fortified with VD [6]. Hepatic and kidney hydroxylation in positions 25 and 1, respectively, are necessary to produce active VD. Kidney 1 alpha hydroxylase is under control of endogenous PTH [38]. VD is crucial for the calcium and skeletal homeostasis in physiological and disease states [39]. It also has many systemic functions which are also known as extraskeletal effects [39], including immunomodulation, most of which are based on solid experimental evidences and positive observational studies. Assessment of total $25 \mathrm{OH}$ VD is widely accepted as a marker of the VD status, with $25 \mathrm{OH}$ VD levels below $12 \mathrm{ng} / \mathrm{mL}$ representing deficiency and levels above $30 \mathrm{ng} / \mathrm{mL}$ sufficiency [40].

Since our letter of March 2020 in which we hypothesized that widespread hypovitaminosis $\mathrm{D}$ could have been responsible for the huge lethality of COVID-19 in Italy [8], several reports corroborated our assumption. In fact, patients with hypovitaminosis $\mathrm{D}$ at time of evaluation were shown to be at increased risk of testing positive for COVID-19 vs patients with sufficient status [41, 42]. Moreover, low 25OHVD levels on hospital admission have been shown also to be associated with COVID-19 disease stage and mortality [43, 44].

\section{Vitamin D and SARS-CoV-2 positivity and COVID-19 severity}

In a recent study from Armenia, slightly less than half of the hospitalized COVID-19 patients showed $25 \mathrm{OH}$ VD < $12 \mathrm{ng} / \mathrm{ml}$, while average levels of VD were lower than those recorded in general population [45]. This correlation was reported to remain strong even after adjusting for potential confounding factors (age, gender, latitude, or ethnicity) [46]. Other studies confirmed lower levels of 25OHVD among COVID-19 patients [47]. Negative relationship between VD status and hospitalization risk, disease severity and biochemical activity as well as a double mortality risk were reported [48]. Interestingly, also in the few studies not confirming the relationship between VD status and SARS-CoV-2 positivity or COVID severity such as those based on UK Biobank ethnicity did show a significant impact at least in univariate analysis [49].

Of note, in another very recent study male patients with COVID-19 showed progressively lower 25OHVD and prevalence of hypovitaminosis D with advancing stage of the disease based on chest CT. Hypovitaminosis D was also significantly associated with mortality (with an odds ratio slightly $<4$ ) independently of other demographical and clinical variables [50].

\section{Vitamin D intervention in COVID-19}

Very few data from controlled studies on role of VD supplementation in prevention and treatment of COVID-19 are available at present despite the relatively strong associative data mentioned above. In a Spanish pilot clinical trial, 76 consecutive patients hospitalized with COVID-19 and pneumonia were randomized to receiving the center-specific standard of care alone or combined with calcifediol. Interestingly, the need of ICU admission was much lower in the VD-treated group (2\%) vs standard treatment (50\%). In this latter group, two patients died vs none in the VD-treated group [51]. Unfortunately, blood 25OHVD values at baseline or during treatment were not available [51]. However, authors did point out that adults living in their specific area of Spain and season of the year are on average VD deficient [51]. Importantly, calcifediol is known to be able to quickly elevate blood 25OHVD levels [52].

\section{Epidemiological evidence linking hypovitaminosis D and COVID-19}

Interestingly, according to epidemiological evidence in Italy, as in other Mediterranean Countries, the prevalence of hypovitaminosis D is one of the highest in Europe. In fact, in elderly Italian women prevalence of VD deficiency was found in about $75 \%$ of them [53].

Change to an indoor lifestyle and lack of food fortification with VD is likely the main reason accounting for the higher prevalence of low circulating VD levels in Italy and in other Mediterranean Countries vs many other central and North European Countries in which this is systemically the rule from several decades due to the low sun exposure leading to what is defined Scandinavian paradox [54, 55]. In fact, a geographical overlap between high prevalence of hypovitaminosis $\mathrm{D}$ and elevated number and severity of COVID-19 cases has been observed [43]. A few other studies reported an association between COVID-19 prevalence and related mortality with latitude and consequent sunlight availability [56]. Importantly, home confinement is the most used measure in order to prevent the spreading of SARS-CoV-2 infection but the drawback of this extremization of the above mentioned progressive changes in 
lifestyle may be the worsening in the VD status in already at huge risk patients particularly in heavily VD-deficient countries [54].

\section{Pathophysiological evidence linking hypovitaminosis $D$ and COVID-19}

Hypovitaminosis D was suggested to be linked to systemic infections and impaired immune response or even autoimmune diseases [39]. Moreover, a recent meta-analysis reported that VD supplementation can prevent respiratory infections [57].

VD is a negative modulator of renin-angiotensinaldosterone system and through ACE2 downregulation may mitigate clinical manifestations of COVID-19 [58]. Moreover, since vitamin D-binding protein (VDBP) [59] may be involved in ARDS [60] through association with actin, CD44, and annexin A2 [61, 62] in the presence of low VD levels in the blood an increased availability of VDBP for complement and neutrophil activation may be observed [63, 64].

VD has also been hypothesized to be key in counteracting the cytokine storm characteristic of severe COVID-19 [6]. Mechanistically, VD may modulate production of proinflammatory cytokines $[65,66]$. Moreover, active VD regulates T-cell differentiation vs Th2 phenotype [67] with antiinflammatory effect in the cytokine storm of COVID-19 [68].

Finally, in severely compromised patients independently from their ICU admission, very low levels of 25OHVD were reported [69] and it was hypothesized that since poor VD status may negatively impact the outcome of ICU patients supplementation with high doses of VD could reduce their morbidity and mortality [70].

\section{Hypovitaminosis D in endocrine comorbidities and conditions associated with COVID-19 severity}

In this section, we will review the evidence that allow to hypothesize that low VD could be the link between age, sex, endocrine comorbidities, and increased susceptibility to severe COVID-19.

\section{Aging and sex}

Aging Advancing age impairs skin ability to synthesize cholecalciferol [71]. Moreover, emphasis on avoiding sun irradiation in order to prevent skin cancer progressively increased [72]. Combination of these factors may cause poorer VD status in older vs younger subjects [73]. In fact, some, but not all, studies showed lower 25OHVD levels with aging [74]. In this regard, a meta-analysis on 33,000 individuals reported that those aged $>75$ years to had lower $25 \mathrm{OHVD}$ vs those aged 65-75 years [75]. However, despite many national surveys did not find huge age-dependent differences in 25OHVD or in prevalence of VD deficiency [76], institutionalized older adults were reported to be at greater risk for VD deficiency [77]. Consequently, the Institute of Medicine, as well as the International Osteoporosis Foundation, recommended higher dietary intake of VD (800-1000 IU/day) for people older than 70 years of age vs that recommended for younger subjects (600 IU/day) [78, 79].

Sex Interestingly, VD is still mainly used in patients with osteoporosis, the vast majority of whom are postmenopausal women. In fact, in a recent study on patients with COVID-19, of the 59\% VD-deficient subjects on admission the majority were males (67\%) [50].

Therefore, it can be hypothesized that greater prevalence of hypovitaminosis $\mathrm{D}$ can be a predisposing factor to major vulnerability to COVID-19 in older men vs older postmenopausal women [80]. Our preliminary data suggested that women older than 50 years under pharmacologic treatment for osteoporosis were not at increased risk of either symptomatic or severe COVID-19 [81]. Interestingly, most of the patients were on VD in combination with bone protective agents [82-84]. Therefore, it was hypothesized that female patients with osteoporosis could have been somewhat protected from COVID by VD as part of the pharmacologic bone sparing treatments.

\section{Diabetes mellitus and diabetic retinopathy}

Diabetes It was widely reported that patients with type 2 diabetes had low 25OHVD levels [85]. Interestingly, in an observational Italian study postmenopausal diabetic women showed significantly greater prevalence of severe VD deficiency vs control subjects (39\% vs $25 \%$, respectively) [86]. In fact, reduced 25OHVD levels were hypothesized to be involved in the pathophysiology of diabetes-related skeletal fragility [87]. Recently, low circulating VD was reported to be associated with poor glycemic control in diabetic patients [88]. Moreover, large prospective studies suggested that low VD may predispose to a higher risk of developing impaired fasting glucose and diabetes [89, 90].

Recent studies and a meta-analysis of randomized trials showed that VD supplementation in diabetes can improve HbA1c, insulin resistance, and insulin secretion [91, 92] as well as fasting glucose particularly when glyco-metabolic control is poor [93].

Some studies also suggest that VD treatment may slow the progression to diabetes in either patients at high risk of diabetes or with prediabetes, specifically in those with low baseline 25OH VD [94]. Very recently, it was also reported that black people with diabetes and COVID-19 are more likely to be hospitalized and die than white ones [95].

Although convincing data on VD levels in the diabetic subgroup of COVID-19 patients in particular are not yet 
available [96], it can be hypothesized that lack of VD may be detrimental or even causal in the bidirectional relationship between diabetes and COVID-19 increasing synergistically the vulnerability of diabetic patients to the infection as well as facilitating the diabetogenic action of COVID-19.

Diabetic retinopathy (DR) An inverse relationship between presence and severity of DR, and VD concentration is well established with lowest levels in proliferative DR and highest in diabetic patients without DR [97]. In crosssectional studies, confirmed by meta-analyses from available observational studies, prevalence of DR was reported to be double in VD-deficient vs VD sufficient patients and DR was associated with VD deficiency. As well, higher prevalence of severe and mild DR in poorly controlled diabetic subjects with low vs normal VD and association between VD deficiency and DR severity (OR: 2.22; 95\% CI: $1.36,3.65)$ was found [98-101]. Interestingly, subanalysis of the Field and Rotterdam prospective studies showed that subjects with hypovitaminosis D had a higher cumulative incidence of microvascular events and were at increased risk for DR, respectively [102, 103].

Pathophysiologically, an association between VD and vascular function has been described in patients with diabetes [104]; moreover, a report described improved vascular function parameters in patients with diabetes, after VD oral supplementation [105]. Furthermore, patients with diabetes and VD deficiency had reduced endotheliumdependent microvascular function [106]. It is noteworthy that VDR is expressed in endothelial cells (EC), pericytes, and vascular smooth muscle cells and suppression of VDR in EC alters vascular homeostasis [107].

Therefore, it can be hypothesized that low VD, which characterizes diabetic patients with retinopathy, can be a common denominator of the microvascular damage in COVID-19 of which, in turn, diabetic retinopathy may be an epiphenomenon.

\section{Obesity and body composition}

Low levels of $25 \mathrm{OH}$ VD were very frequently found in obese nondiabetic particularly elderly subjects being inversely related to BMI and adiposity [73, 108], with negative effects on bone and muscle health. Interestingly, VD deficiency was also reported to be associated with decreased muscle mass and function thereby predisposing to an obese osteosarcopenic phenotype which is also observed in patients with prostate or breast cancer under medical treatment [109, 110].

Therefore, VD may be considered key for maintaining of an healthy body composition protecting from obesity and sarcopenia. However, only a few and generally not adequately powered randomized trials tested efficacy of VD supplementation on weight loss in obese subjects [111]. A recent meta-analysis which included more than 17,000 persons showed that circulating VD was inversely associated with body fat mass [112]. Interestingly, resistance to VD in overweight and obesity was recently reported in hypoparathyroid patients [113].

Pathophysiologically, despite the above reported epidemiological evidence the mechanisms underlying association between VD and obesity are still debated. Interestingly, it has been suggested that VD may exert a protective effect in obese individuals, by reducing systemic inflammation [114]. Adipose tissue is a direct target of VD, as confirmed by the presence of VDR in both subcutaneous and visceral adipocytes. In fact, VD has been suggested to play a role in modulating fat distribution and activity [115].

A direct relationship between VD status, body fat, age, and SARS-CoV-2 infection and COVID-19 outcomes has been suggested. In fact, fat accumulates with aging, and sequestrating active VD decreases its bioavailability and action [116]. Therefore, very low VD levels in obese subjects may be associated with more severe COVID-19 and eventually worsen the prognosis in those subjects admitted to ICU due to enhanced baseline inflammatory state. Low VD levels may also play an important role in the preservation of body composition also in the recovery phase post COVID-19.

\section{Vertebral fractures}

Low VD has been traditionally linked with low bone mass and increased risk of fractures. The VD-related increase in BMD of the hip was observed to be larger in young vs older adults [117]. Two recent randomized trials confirmed a positive effect of VD supplementation on BMD in subjects with low baseline VD [118, 119]. Several long-term studies also found an association between poor VD status and fractures in elderly people [120]. Also, a few trials reported that VD either alone or more effectively when combined with calcium determined a significant decrease of fracture incidence [121-124].

Overall, the available meta-analyses showed that the bone protective effects of VD were greater in institutionalized VD-deficient older subjects at sufficiently large dose (at least 800 IU/day) [125]. The Cochrane systematic review and meta-analysis showed that combined VD and calcium supplementation may obtain a decrease in hip fracture and non-VF risk around 15\% [126]. In a Chinese study, women with morphometric VFs and hypovitaminosis D had higher PTH levels and more frequently secondary hyperparathyroidism as compared to fracture-free controls [127]. No trial/meta-analysis is available on VD and VFs but it can well be hypothesized that those COVID-19 patients by us observed bearing VFs at hospital admission could have been predisposed to these skeletal events by low levels of VD. 


\section{Hypocalcemia}

Active VD acts on the small bowel to increase intestinal calcium absorption. VD deficiency leads to a compensatory increase in PTH to maintain calcium levels with the clinically relevant exception of patients with hypoparathyroidism [128-130]. Severe forms of VD deficiency can cause hypocalcemia and rickets/osteomalacia. Milder forms of VD insufficiency also can negatively affect bone via secondary hyperparathyroidism [39].

Interestingly, several studies showed that not only in patients with postsurgical hypoparathyroidism [26] but also in cases of blunted PTH secretion or impaired compensatory feedback mechanism low levels of VD may be associated with hypocalcemia. In fact, in prospective studies on patients undergoing thyroid surgery, positive correlation between preoperative VD deficiency and postoperative hypocalcemia is not necessarily linked to low PTH [131].

In fact, since intestinal calcium absorption is impaired in VD-deficient subjects, maintenance of eucalcemia depends mainly on the compensatory increase in PTH level, which mediates bone and renal calcium reabsorption. In the case typically observed in the post-thyroidectomy period of blunted PTH compensatory response even mildly vitamin D deficient patients can present severe hypocalcemia. Recently, no differences in PTH levels between VD-deficient and sufficient COVID-19 patients were observed [132] pointing toward an hypothesis of possibly impaired PTH compensatory response in such patients which, in turn, might result in hypocalcemia in pre-COVID-19 VD-deficient subjects

It has also been previously reported that particularly in COVID-19 elevated levels of pro-inflammatory unbound and unsaturated fatty acids (UFA) may cause hypocalcemia binding calcium [133].

Pathophysiologically, it is unlikely that one single mechanism could be able to explain a widespread finding such as hypocalcemia in a quite heterogeneous COVID-19 population with different degrees of severity and clinical manifestations [5]. In fact, we can hypothesize that low VD may act in synergism with factors directly predisposing to hypocalcemia such as calcium dependent viral mechanisms of action, enhanced cellular permeability to calcium ions and the increase in UFA as well with other still unexplored indirect mechanisms leading to a possibly blunted PTH response to hypocalcemia [134].

\section{Conclusions}

Hypovitaminosis D is a dangerous widespread condition particularly in the time of COVID-19 pandemic. In fact, it could be directly linked to an increased predisposition to SARS-CoV-2 infection as well as to an increased severity of COVID-19. This negative effect may be exerted directly through the lack of immunomodulating and vasoprotective actions of VD. Importantly, we have shown in this piece that most of the known conditions and endocrine comorbidities which associate with severe outcomes of COVID19 are characterized or caused by low VD levels. Although data clearly relating low VD levels to the negative impact of these comorbidities are largely lacking, it seems reasonable to grant through appropriate supplementation an adequate level of VD to all patients bearing these comorbidities in a clinically sound, and largely safe, attempt to improve the endocrine phenotype of COVID-19.

\section{Compliance with ethical standards}

Conflict of interest The author declares no competing interests.

Publisher's note Springer Nature remains neutral with regard to jurisdictional claims in published maps and institutional affiliations.

\section{References}

1. A. Gupta, M.V. Madhavan, K. Sehgal, N. Nair, S. Mahajan, T.S. Sehrawat, B. Bikdeli, N. Ahluwalia, J.C. Ausiello, E.Y. Wan, D. E. Freedberg, A.J. Kirtane, S.A. Parikh, M.S. Maurer, A.S. Nordvig, D. Accili, J.M. Bathon, S. Mohan, K.A. Bauer, M.B. Leon, H.M. Krumholz, N. Uriel, M.R. Mehra, M.S.V. Elkind, G. W. Stone, A. Schwartz, D.D. Ho, J.P. Bilezikian, D.W. Landry, Extrapulmonary manifestations of COVID-19. Nat. Med. 26, 1017-1032 (2020). https://doi.org/10.1038/s41591-020-0968-3

2. J. Lan et al., Structure of the SARS-CoV-2 spike receptor-binding domain bound to the ACE2 receptor. Nature 581, 215-220 (2020)

3. Ackermann, M. et al., Pulmonary vascular endothelialitis, thrombosis, and angiogenesis in Covid-19. N. Engl. J. Med. (2020). https://doi.org/10.1056/NEJMoa2015432

4. M. Puig-Domingo, M. Marazuela, A. Giustina, COVID-19 and endocrine diseases. A statement from the European Society of Endocrinology. Endocrine 68, 2-5 (2020). https://doi.org/10. 1007/s12020-020-02294-5

5. M. Marazuela, A. Giustina, M. Puig-Domingo, Endocrine and metabolic aspects of the COVID-19 pandemic. Rev. Endocr. Metab. Disord. 21, 495-507 (2020). https://doi.org/10.1007/ s11154-020-09569-2

6. J.P. Bilezikian, D. Bikle, M. Hewison, M. Lazaretti-Castro, A.M. Formenti, A. Gupta, M.V. Madhavan, N. Nair, V. Babalyan, N. Hutchings, N. Napoli, D. Accili, N. Binkley, D.W. Landry, A. Giustina, Mechanisms in endocrinology: vitamin D and COVID19. Eur. J. Endocrinol. 183, R133-R147 (2020). https://doi.org/ 10.1530/EJE-20-0665

7. M.L. Brandi, A. Giustina, Sexual dimorphism of coronavirus 19 morbidity and lethality. Trends Endocrinol. Metab. 31, 918-927 (2020). https://doi.org/10.1016/j.tem.2020.09.003

8. A. Giustina, A.M. Formenti, Preventing a covid-19 pandemic Can high prevalence of severe hypovitaminosis D play a role in the high impact of Covid infection in Italy? BMJ 368, m810 (2020)

9. F. Rubino, S.A. Amiel, P. Zimmet, G. Alberti, S. Bornstein, R.H. Eckel, G. Mingrone, B. Boehm, M.E. Cooper, Z. Chai, S. Del Prato, L. Ji, D. Hopkins, W.H. Herman, K. Khunti, J.C. Mbanya, E. Renard, New-onset diabetes in Covid-19. N. Engl. J. Med. 383, 789-790 (2020). https://doi.org/10.1056/NEJMc2018688 
10. M.M. Lima-Martínez, C. Carrera Boada, M.D. Madera-Silva, W. Marín, M. Contreras, COVID-19 and diabetes: a bidirectional relationship. Clin. Investig. Arterioscler. S0214-9168, 30105-4 (2020). https://doi.org/10.1016/j.arteri.2020.10.001

11. M. Martins, J.M. Boavida, J.F. Raposo, F. Froes, B. Nunes, R.T. Ribeiro, M.P. Macedo, C. Penha-Gonçalves, Diabetes hinders community-acquired pneumonia outcomes in hospitalized patients. BMJ Open Diabetes Res. Care 4, e000181 (2016). https://doi.org/10.1136/bmjdrc-2015-000181

12. M. Apicella, M.C. Campopiano, M. Mantuano, L. Mazoni, A. Coppelli, S. Del Prato, COVID-19 in people with diabetes: understanding the reasons for worse outcomes. Lancet Diabetes Endocrinol. 8, 782-792 (2020). https://doi.org/10.1016/S22138587(20)30238-2

13. B. Cariou, S. Hadjadj, M. Wargny, M. Pichelin, A. Al-Salameh, I. Allix et al., Phenotypic characteristics and prognosis of inpatients with COVID-19 and diabetes: the CORONADO study. Diabetologia 63, 1500-1515 (2020)

14. A. Corcillo, S. Cohen, A. Li, J. Crane, D. Kariyawasam, J. Karalliedde, Diabetic retinopathy is independently associated with increased risk of intubation: a single centre cohort study of patients with diabetes hospitalised with COVID-19. Diabetes Res. Clin. Pract. 108529 (2020). https://doi.org/10.1016/j.dia bres.2020.108529

15. L.M. Donini, L. Busetto, J.M. Bauer, S. Bischoff, Y. Boirie, T. Cederholm, A.J. Cruz-Jentoft, D. Dicker, G. Frühbeck, A. Giustina, M.C. Gonzalez, H.S. Han, S.B. Heymsfield, T. Higashiguchi, A. Laviano, A. Lenzi, E. Parrinello, E. Poggiogalle, C. M. Prado, J.S. Rodriguez, Y. Rolland, F. Santini, M. Siervo, F. Tecilazich, R. Vettor, J. Yu, M. Zamboni, R. Barazzoni, Critical appraisal of definitions and diagnostic criteria for sarcopenic obesity based on a systematic review. Clin. Nutr. 39, 2368-2388 (2020). https://doi.org/10.1016/j.clnu.2019.11.024

16. C. Welch, C. Greig, T. Masud, D. Wilson, T.A. Jackson, COVID-19 and acute sarcopenia. Aging Dis. 11, 1345-1351 (2020). https://doi.org/10.14336/AD.2020.1014

17. A. Simonnet, M. Chetboun, J. Poissy, V. Raverdy, J. Noulette, A. Duhamel et al., High prevalence f obesity in severe acute respiratory syndrome coronavirus-2 (SARS-CoV-2) requiring invasive mechanical ventilation. Obesity (2020). https://doi.org/ 10.1002/oby. 22831

18. Y. Huang, Y. Lu, Y.M. Huang, M. Wang, W. Ling, Y. Sui, H.L. Zhao, Obesity in patients with COVID-19: a systematic review and meta-analysis. Metabolism 113, 154378 (2020). https://doi. org/10.1016/j.metabol.2020.154378

19. M.V. Davi', L. Dalle Carbonare, A. Giustina, M. Ferrari, A. Frigo, V. Lo Cascio, G. Francia, Sleep apnoea syndrome is highly prevalent in acromegaly and only partially reversible after biochemical control of the disease. Eur. J. Endocrinol. 159, 533-540 (2008). https://doi.org/10.1530/EJE-08-0442

20. J.M. Brock, A. Billeter, B.P. Müller-Stich, F. Herth, Obesity and the lung: what we know today. Respiration 1-11 (2020). https:// doi.org/10.1159/000509735

21. M. Castellana, E. Conte, A. Cignarelli, S. Perrini, A. Giustina, L. Giovanella, F. Giorgino, P. Trimboli, Efficacy and safety of very low calorie ketogenic diet (VLCKD) in patients with overweight and obesity: a systematic review and meta-analysis. Rev. Endocr. Metab. Disord. 21, 5-16 (2020). https://doi.org/10.1007/s11154019-09514-y

22. V.J. Vieira-Potter, Inflammation and macrophage modulation in adipose tissues. Cell Microbiol. 16, 1484-1492 (2014). https:// doi.org/10.1111/cmi.12336

23. N. Sattar, I.B. McInnes, J.J.V. McMurray, Obesity is a Risk Factor for Severe COVID-19 Infection: Multiple Potential Mechanisms. Circulation 142(1), 4-6 (2020). https://doi.org/ 10.1161/CIRCULATIONAHA.120.047659
24. L. di Filippo, A.M. Formenti, M. Doga, E. Pedone, P. RovereQuerini, A. Giustina, Radiological thoracic vertebral fractures are highly prevalent in COVID-19 and predict disease otcomes. J. Clin. Endocrinol. Metab. dgaa738 (2020). https://doi.org/10. 1210/clinem/dgaa738

25. S. Frara, M. Losa, M. Doga, A.M. Formenti, P. Mortini, G. Mazziotti, A. Giustina, High prevalence of radiological vertebral fractures in patients with TSH-secreting pituitary adenoma. J. Endocr. Soc. 2, 1089-1099 (2018). https://doi.org/10.1210/js. 2018-00091

26. A.M. Formenti, F. Tecilazich, R. Giubbini, A. Giustina, Risk of vertebral fractures in hypoparathyroidism. Rev. Endocr. Metab. Disord. 20, 295-302 (2019). https://doi.org/10.1007/ s11154-019-09507-x

27. S. Bossoni, L. Chiesa, A. Giustina, Severe hypocalcemia in a thyroidectomized woman with Covid-19 infection. Endocrine 68, 253-254 (2020)

28. L. Di Filippo, A.M. Formenti, P. Rovere-Querini et al., Hypocalcemia is highly prevalent and predicts hospitalization in patients with COVID-19. Endocrine 68, 475-478 (2020)

29. L. di Filippo, A.M. Formenti, M. Doga, S. Frara, P. RovereQuerini, E. Bosi, M. Carlucci, A. Giustina, Hypocalcemia is a distinctive biochemical feature of hospitalized COVID-19 patients. Endocrine. 1-5 (2020). https://doi.org/10.1007/ s12020-020-02541-9

30. F. Cappellini, R. Brivio, M. Casati, A. Cavallero, E. Contro, P. Brambilla, Brambilla, Low levels of total and ionized calcium in blood of COVID-19 patients. Clin. Chem. Lab. Med. 58(9), e171-e173 (2020).

31. J. Liu, P. Han, J. Wu, J. Gong, D. Tian, Prevalence and predictive value of hypocalcemia in severe COVID-19 patients. J. Infect. Public Health 13(9), 1224-1228 (2020). https://doi.org/ 10.1016/j.jiph.2020.05.029

32. J.K. Sun, W.H. Zhang, L. Zou et al., Serum calcium as a biomarker of clinical severity and prognosis in patients with coronavirus disease 2019. Aging 12(12), 11287-11295 (2020). https://doi.org/10.18632/aging.103526

33. J.K. Millet et al., Physiological and molecular triggers for SARS$\mathrm{CoV}$ membrane fusion and entry into host cells. Virology 517, 3-8 (2018)

34. M.R. Straus et al., $\mathrm{Ca}^{2+}$ ions promote fusion of Middle East respiratory syndrome coronavirus with host cells and increase infectivity. J. Virol. https://doi.org/10.1128/JVI.00426-20 (2020)

35. L. Nathan et al., Calcium ions directly interact with the Ebola virus fusion peptide to promote structure-function changes that enhance infection. ACS Infect. Dis. 6, 250-260 (2020)

36. C.M. Booth et al., Clinical features and short-term outcomes of 144 patients with SARS in the Greater Toronto Area. JAMA 289, 2801-2809 (2003)

37. T.M. Uyeki et al., Clinical management of Ebola virus disease in the United States and Europe. N. Engl. J. Med. 374, 636-646 (2016)

38. G. Mazziotti, A.M. Formenti, R.A. Adler, J.P. Bilezikian, A. Grossman, E. Sbardella, S. Minisola, A. Giustina, Glucocorticoid-induced osteoporosis: pathophysiological role of GH/IGF-I and PTH/VITAMIN D axes, treatment options and guidelines. Endocrine 54, 603-611 (2016). https://doi.org/10. 1007/s12020-016-1146-8

39. R. Bouillon, C. Marcocci, G. Carmeliet et al., Skeletal and extraskeletal actions of vitamin D: current evidence and outstanding questions. Endocr. Rev. 40, 1109-1151 (2019)

40. C.T. Sempos, A.C. Heijboer, D.D. Bikle, J. Bollerslev, R. Bouillon, P.M. Brannon, H.F. DeLuca, G. Jones, C.F. Munns, J. P. Bilezikian, A. Giustina, N. Binkley, Vitamin D assays and the definition of hypovitaminosis D: results from the First International Conference on Controversies in Vitamin D. Br. J. Clin. 
Pharmacol. 84, 2194-2207 (2018). https://doi.org/10.1111/bcp. 13652

41. D.O. Meltzer, T.J. Best, H. Zhang, T. Vokes, V. Arora, J. Solway, Association of vitamin D status and other clinical characteristics with COVID-19 test results. JAMA Netw. Open 3, e2019722 (2020). https://doi.org/10.1001/jamanetworkopen. 2020.19722

42. N. Hutchings, V. Babalyan, S. Baghdasaryan, M. Qefoyan, N. Sargsyants, E. Aghajanova, A. Martirosyan, R. Harutyunyan, O. Lesnyak, A.M. Formenti, A. Giustina, J.P. Bilezikian, Patients hospitalized with COVID-19 have low levels of 25-hydroxyvitamin D. Endocrine (2021). https://doi.org/10.1007/s12020-020-02597-7

43. L.L. Benskin, A basic review of the preliminary evidence that COVID-19 risk and severity is increased in vitamin D deficiency. Front. Public Health 8, 513 (2020). https://doi.org/10.3389/ fpubh.2020.00513

44. D. Ferrari, M. Locatelli, M. Briguglio, G. Lombardi, Is there a link between vitamin D status, SARS-CoV-2 infection risk and COVID-19 severity? Cell Biochem. Funct. (2020). https://doi. org/10.1002/cbf.3597

45. N. Hutchings, V. Babalyan, A. Heijboer et al., Vitamin D insufficiency is widespread in Armenia. ASBMR 2020 Annual meeting virtual event Poster Sessions, Presentation Number: P592 www.asbmr.org. Poster Presentation (American Society for Bone and Mineral Research Annual Meeting, 2020)

46. E. Merzon, D. Tworowski, A. Gorohovski et al., Low plasma 25 $(\mathrm{OH})$ vitamin $\mathrm{D}$ level is associated with increased risk of COVID19 infection: an Israeli population-based study. FEBS J. 17, 3693-3702 (2020)

47. Z. Maghbooli, M.A. Sahraian, M. Ebrahimi et al., Vitamin D sufficiency, a serum 25-hydroxyvitamin D at least $30 \mathrm{ng} / \mathrm{mL}$ reduced risk for adverse clinical outcomes in patients with COVID-19 infection. PLoS ONE 15(9), e0239799 (2020)

48. H.W. Kaufman, J.K. Niles, M.H. Kroll, C. Bi, M.F. Holick, SARS-CoV-2 positivity rates associated with circulating 25hydroxyvitamin D levels. PLoS ONE 15(9), e0239252 (2020)

49. C.E. Hastie, D.F. Mackay, F. Ho et al., Vitamin D concentrations and COVID-19 infection in UKbiobank. Diabetes Metab. Syndr. 14(4), 561-56 (2020)

50. D. De Smet, K. De Smet, P. Herroelen, S. Gryspeerdt, G.A. Martens, Serum 25(OH)D level on hospital admission associated with COVID-19 stage and mortality. Am. J. Clin. Pathol. aqaa252 (2020). https://doi.org/10.1093/ajcp/aqaa252

51. M. Entrenas Castillo, L.M. Entrenas Costa, J.M. Vaquero Barrios, J.F. Alcalá Díaz, J. López Miranda, R. Bouillon, J.M. Quesada Gomez, "Effect of calcifediol treatment and best available therapy versus best available therapy on intensive care unit admission and mortality among patients hospitalized for COVID-19: A pilot randomized clinical study". J. Steroid Biochem. Mol. Biol. 203, 105751 (2020). https://doi.org/10.1016/j. jsbmb.2020.105751

52. A. Giustina, R.A. Adler, N. Binkley, J. Bollerslev, R. Bouillon, B. Dawson-Hughes, P.R. Ebeling, D. Feldman, A.M. Formenti, M. Lazaretti-Castro, C. Marcocci, R. Rizzoli, C.T. Sempos, J.P. Bilezikian, Consensus statement from $2^{\text {nd }}$ international conference on controversies in vitamin D. Rev. Endocr. Metab. Disord. 21, 89-116 (2020). https://doi.org/10.1007/s11154-01909532-w

53. G. Isaia, R. Giorgino, G.B. Rini, M. Bevilacqua, D. Maugeri, S. Adami, Prevalence of hypovitaminosis D in elderly women in Italy: clinical consequences and risk factors. Osteoporos. Int. 14, 577-582 (2003)

54. A. Giustina, R. Bouillon, N. Binkley, C. Sempos, R.A. Adler, J. Bollerslev, B. Dawson-Hughes, P.R. Ebeling, D. Feldman, A. Heijboer, G. Jones, C.S. Kovacs, M. Lazaretti-Castro, P. Lips, C. Marcocci, S. Minisola, N. Napoli, R. Rizzoli, R. Scragg, J.H.
White, A.M. Formenti, J.P. Bilezikian, Controversies in vitamin D: a statement from the third international conference. JBMR 4, e10417 (2020). https://doi.org/10.1002/jbm4.10417

55. A. Giustina, R.A. Adler, N. Binkley et al., Controversies in vitamin D: summary statement from an international conference. J. Clin. Endocrinol. Metab. 104, 234-240 (2019)

56. P.B. Whittemore, COVID-19 fatalities, latitude, sunlight, and vitamin D. Am. J. Infect. Control 48(9), 1042-1044 (2020)

57. A.R. Martineau, D.A. Jolliffe, R.L. Hooper et al., Vitamin D supplementation to prevent acute respiratory tract infections: systematic review and meta-analysis of individual participant data. BMJ 356, i6583 (2017)

58. J.A. Grace, S. Klein, C.B. Herath et al., Activation of the MAS receptor by angiotensin-(1-7) in the renin-angiotensin system mediates mesenteric vasodilatation in cirrhosis. Gastroenterology 145(4), 874-884 (2013)

59. G. Mazziotti, F. Maffezzoni, A. Giustina, Vitamin D-binding protein: one more piece in the puzzle of acromegalic osteopathy? Endocrine 52, 183-186 (2016). https://doi.org/10.1007/s12020016-0890-0

60. M.M. Speeckaert, J.R. Delanghe, Association between low vitamin D and COVID-19: don't forget the vitamin D binding protein. Aging Clin. Exp. Res. 32(7), 1207-1208 (2020)

61. M.M. Speeckaert, R. Speeckaert, N. van Geel, J.R. Delanghe, Vitamin D binding protein: a multifunctional protein of clinical importance. Adv. Clin. Chem. 63, 1-57 (2014)

62. L. Du, J. Zhou, J. Zhang et al., Actin filament reorganization is a key step in lung inflammation induced by systemic inflammatory response syndrome. Am. J. Respir. Cell Mol. Biol. 47(5), 597-603 (2012)

63. R.R. Kew, The vitamin D binding protein and inflammatory injury: a mediator or sentinel of tissue damage? Front. Endocrinol. 10, 470 (2019)

64. L.E. Gralinski, T.P. Sheahan, T.E. Morrison et al., Complement activation contributes to severe acute respiratory syndrome coronavirus pathogenesis. mBio 9(5), 1-15 (2018)

65. P. Mehta, D.F. McAuley, M. Brown et al., COVID-19: consider cytokine storm syndromes and immunosuppression. Lancet 395 (10229), 1033-1034 (2020)

66. X. Chen, B. Zhao, Y. Qu et al., Detectable serum SARS-CoV-2 viral load (RNAaemia) is closely correlated with drastically elevated interleukin 6 (IL-6) level in critically ill COVID-19 patients. Clin. Infect. Dis. (2020). https://doi.org/10.1093/cid/ ciaa449

67. D.D. Bikle, J. Schwartz, Vitamin D binding protein, total and free vitamin D levels in different physiological and pathophysiological conditions. Front. Endocrinol. 10, 317 (2019)

68. N. Vyas, S.J. Kurian, D. Bagchi et al., Vitamin D in prevention and treatment of COVID-19: current perspective and future prospects. J. Am. Coll. Nutr. 1-14 (2020). https://doi.org/10. 1080/07315724.2020.1806758

69. K. Amrein, B. Venkatesh, Vitamin D and the critically ill patient. Curr. Opin. Clin. Nutr. Metab. Care 15(2), 188-193 (2012)

70. K.B. Christopher, Vitamin D and critical illness outcomes. Curr. Opin. Crit. Care 22(4), 332-338 (2016)

71. J. MacLaughlin, M.F. Holick, Aging decreases the capacity of human skin to produce vitamin D3. J. Clin. Investig. 76(4), 1536-8 (1985)

72. S. Garcovich, G. Colloca, P. Sollena, B. Andrea, L. Balducci, W. C. Cho et al., Skin cancer epidemics in the elderly as an emerging issue in geriatric oncology. Aging Dis. 8(5), 643-661 (2017)

73. A.A. Ginde, M.C. Liu, C.A. Camargo, Demographic differences and trends of vitamin D insufficiency in the US population, 1988-2004. Arch. Intern. Med. 169(6), 626-632 (2009)

74. J.C. Gallagher, Vitamin D and aging. Endocrinol. Metab. Clin. N. Am. 42(2), 319-332 (2013) 
75. T. Hagenau, R. Vest, T.N. Gissel, C.S. Poulsen, M. Erlandsen, L. Mosekilde et al., Global vitamin D levels in relation to age, gender, skin pigmentation and latitude: an ecologic metaregression analysis. Osteoporos. Int. 20(1), 133-140 (2009)

76. K.D. Cashman, S. Muldowney, B. McNulty, A. Nugent, A.P. FitzGerald, M. Kiely et al., Vitamin D status of Irish adults: findings from the National Adult Nutrition Survey. Br. J. Nutr. 109(7), 1248-1256 (2013)

77. P. Lips, Vitamin D deficiency and secondary hyperparathyroidism in the elderly: consequences for bone loss and fractures and therapeutic implications. Endocr. Rev. 22(4), 477-501 (2001)

78. A.C. Ross, J.E. Manson, S.A. Abrams, J.F. Aloia, P.M. Brannon, S.K. Clinton et al., The 2011 report on dietary reference intakes for calcium and vitamin D from the Institute of Medicine: what clinicians need to know. J. Clin. Endocrinol. Metab. 96(1), 53-58 (2011)

79. B. Dawson-Hughes, A. Mithal, J.-P. Bonjour, S. Boonen, P. Burckhardt, G.E.-H. Fuleihan et al., IOF position statement: vitamin $\mathrm{D}$ recommendations for older adults. Osteoporos. Int 21 (7), 1151-1154 (2010)

80. E. Orwoll et al., Vitamin D deficiency in older men. J. Clin. Endocrinol. Metab. 94, 1214-1222 (2009)

81. A.M. Formenti, E. Pedone, L. di Filippo, F.M. Ulivieri, A. Giustina, Are women with osteoporosis treated with denosumab at risk of severe COVID-19? Endocrine 70, 203-205 (2020). https://doi.org/10.1007/s12020-020-02500-4

82. P.R. Ebeling, R.A. Adler, G. Jones et al., Management of endocrine disease: therapeutics of vitamin D. Eur. J. Endocrinol. 179, R239-R259 (2018)

83. E. Canalis, A. Giustina, J.P. Bilezikian, Mechanisms of anabolic therapies for osteoporosis. N. Engl. J. Med. 357, 905-916 (2007). https://doi.org/10.1056/NEJMra067395

84. M. Doga, S. Bonadonna, A. Burattin, R. Carpinteri, F. Manelli, A. Giustina, Bisphosphonates in the treatment of glucocorticoidinduced osteoporosis. Front. Horm. Res. 30, 150-164 (2002). https://doi.org/10.1159/000061082

85. P. Pietschmann, G. Schernthaner, W. Woloszczuk, Serum osteocalcin levels in diabetes mellitus: analysis of the type of diabetes and microvascular complications. Diabetologia 31, 892-895 (1988)

86. G. Isaia et al., High prevalence of hypovitaminosis D in female type 2 diabetic population. Diabetes Care 24, 1496 (2001)

87. G. Mazziotti, J. Bilezikian, E. Canalis, D. Cocchi, A. Giustina, New understanding and treatments for osteoporosis. Endocrine 41(1), 58-69 (2012). https://doi.org/10.1007/s12020-011-9570-2

88. K.K. Al Dossari, G. Ahmad, A. Aljowair et al., Association of vitamin d with glycemic control in Saudi patients with type 2 diabetes: a retrospective chart review study in an emerging university hospital. J. Clin. Lab. Anal. 34(2), e23048 (2020). https://doi.org/10.1002/jcla.23048

89. S. Afzal et al., Low vitamin D and risk of type 2 diabetes: a prospective cohort study and metaanalysis. Clin. Chem. 59, 381-391 (2013)

90. A. Tsur et al., Decreased serum concentrations of 25hydroxycholecalciferol are associated with increased risk of progression to impaired fasting glucose and diabetes. Diabetes Care 36, 1361-1367 (2013)

91. P. Lemieux et al., Effects of 6-month vitamin D supplementation on insulin sensitivity and secretion: a randomised, placebocontrolled trial. Eur. J. Endocrinol. 181, 287-299 (2019)

92. Z. Hu, J. Chen, X. Sun, L. Wang, A. Wang, Efficacy of vitamin D supplementation on glycemic control in type 2 diabetes patients: a meta-analysis of interventional studies. Medicine 98(14), e14970 (2019). https://doi.org/10.1097/MD.0000000000014970

93. Y.H. Krul-Poel, M.M. Ter Wee, P. Lips, S. Simsek, Management of endocrine disease: the effect of vitamin D supplementation on glycaemic control in patients with type 2 diabetes mellitus: a systematic review and meta-analysis. Eur. J. Endocrinol. 176(1), R1-R14 (2017). https://doi.org/10.1530/EJE-16-0391

94. A.G. Pittas, B. Dawson-Hughes, P. Sheehan et al., Vitamin D supplementation and prevention of type 2 diabetes. N. Engl. J. Med. 381(6), 520-530 (2019). https://doi.org/10.1056/NEJMoa 1900906

95. E.G. Price-Haywood, J. Burton, D. Fort, L. Seoane, Hospitalization and mortality among black patients and white patients with Covid-19. N. Engl. J. Med. 382, 2534-2543 (2020). https:// doi.org/10.1056/NEJMsa2011686

96. S.K. Singh, R. Jain, S. Singh, Vitamin D deficiency in patients with diabetes and COVID- 19 infection. Diabetes Metab. Syndr. 87, 1033-1035 (2020). https://doi.org/10.1016/j.dsx.2020.06.071

97. F. Tecilazich, A.M. Formenti, A. Giustina, Role of vitamin D in diabetic retinopathy: pathophysiological and clinical aspects. Rev. Endocr. Metab. Disord. 1-13 (2020). https://doi.org/10. 1007/s11154-020-09575-4

98. H. Kaur et al., Vitamin D deficiency is associated with retinopathy in children and adolescents with type 1 diabetes. Diabetes Care 34, 1400-1402 (2011)

99. M. Long et al., Glycated hemoglobin A1C and vitamin D and their association with diabetic retinopathy severity. Nutr. Diabetes 7, e281 (2017)

100. B.A. Luo, F. Gao, L.L. Qin, The Association between Vitamin D Deficiency and Diabetic Retinopathy in Type 2 Diabetes: A Meta-Analysis of Observational Studies. Nutrients 9(3), 307 (2017). https://doi.org/10.3390/nu9030307

101. J. Zhang et al., Relationship between vitamin D deficiency and diabetic retinopathy: a meta-analysis. Can. J. Ophthalmol. 52 (Suppl 1), S39-S44 (2017)

102. A. Keech et al., Effects of long-term fenofibrate therapy on cardiovascular events in 9795 people with type 2 diabetes mellitus (the FIELD study): randomised controlled trial. Lancet $\mathbf{3 6 6}$, 1849-1861 (2005)

103. U. Mutlu et al., Vitamin D and retinal microvascular damage: the Rotterdam Study. Medicine 95, e5477 (2016)

104. P. Jha et al., Low serum vitamin D levels are associated with increased arterial stiffness in youth with type 2 diabetes. Diabetes Care 38, 1551-1557 (2015)

105. J.A. Sugden et al., Vitamin D improves endothelial function in patients with type 2 diabetes mellitus and low vitamin D levels. Diabet. Med. 25, 320-325 (2008)

106. S. Munisamy et al., Impaired microvascular endothelial function in vitamin D-deficient diabetic nephropathy patients. J. Cardiovasc. Med. 14, 466-471 (2013)

107. F. Uberti et al., Vitamin D protects human endothelial cells from oxidative stress through the autophagic and survival pathways. J. Clin. Endocrinol. Metab. 99, 1367-1374 (2014)

108. M. Pereira-Santos, P.R.F. Costa, A.M.O. Assis, C.A.S.T. Santos, D.B. Santos, Obesity and vitamin D deficiency: a systematic review and meta-analysis. Obes. Rev. 16(4), 341-349 (2015)

109. A.M. Formenti, A. Dalla Volta, L. di Filippo, A. Berruti, A. Giustina, Effects of Medical Treatment of Prostate Cancer on Bone Health. Trends Endocrinol. Metab. 32(3), 135-158 (2021). https://doi.org/10.1016/j.tem.2020.12.004

110. S. Monteverdi, R. Pedersini, F. Gallo, F. Maffezzoni, A. Dalla Volta, P. Di Mauro, A. Turla, L. Vassalli, M. Ardine, A.M. Formenti, E.L. Simoncini, A. Giustina, R. Maroldi, V. Amoroso, A. Berruti, The Interaction of Lean Body Mass With Fat Body Mass is Associated With Vertebral Fracture Prevalence in Women With Early Breast Cancer Undergoing Aromatase Inhibitor Therapy. JBMR Plus 5(2), e10440 (2020). https://doi. org/10.1002/jbm4.10440

111. A. Bassatne, M. Chakhtoura, R. Saad, G.E. Fuleihan, Vitamin D supplementation in obesity and during weight loss: a review of 
randomized controlled trials. Metabolism 92, 193-205 (2019). https://doi.org/10.1016/j.metabol.2018.12.010

112. E. Hyppönen, B.J. Boucher, Adiposity, vitamin D requirements, and clinical implications for obesity-related metabolic abnormalities. Nutr. Rev. 76(9), 678-692 (2018). https://doi.org/10. 1093/nutrit/nuy034

113. A.M. Formenti, F. Tecilazich, S. Frara, R. Giubbini, H. De Luca, A. Giustina, Body mass index predicts resistance to active vitamin D in patients with hypoparathyroidism. Endocrine 66, 699-700 (2019)

114. C.P. Earthman, L.M. Beckman, K. Masodkar, S.D. Sibley, The link between obesity and low circulating 25-hydroxyvitamin D concentrations: considerations and implications. Int J. Obes. 36 (3), 387-396 (2012)

115. C. Ding, D. Gao, J. Wilding, P. Trayhurn, C. Bing, Vitamin D signalling in adipose tissue. Br. J. Nutr. 108(11), 1915-1923 (2012)

116. H.K. Biesalski, Obesity, vitamin D deficiency and old age a serious combination with respect to coronavirus disease-2019 severity and outcome. Curr. Opin. Clin. Nutr. Metabol. Care (2020). https://doi.org/10.1097/mco.0000000000000700

117. H.A. Bischoff-Ferrari, T. Dietrich, E.J. Orav, B. DawsonHughes, Positive association between 25-hydroxy vitamin D levels and bone mineral density: a population-based study of younger and older adults. Am. J. Med. 116(9), 634-639 (2004)

118. H.M. Macdonald, I.R. Reid, G.D. Gamble, W.D. Fraser, J.C. Tang, A.D. Wood, 25-hydroxyvitamin D threshold for the effects of vitamin D supplements on bone density: secondary analysis of a randomized controlled trial. J. Bone Miner. Res. 33(8), 1464-1469 (2018)

119. I.R. Reid, A.M. Horne, B. Mihov, G.D. Gamble, F. Al-Abuwsi, M. Singh, L. Taylor, S. Fenwick, C.A. Camargo, A.W. Stewart, R. Scragg, Effect of monthly high-dose vitamin D on bone density in community-dwelling older adults substudy of a randomized controlled trial. J. Intern. Med. 282(5), 452-460 (2017)

120. R. Bouillon, N.M. Van Schoor, E. Gielen, S. Boonen, C. Mathieu, D. Vanderschueren, P. Lips, Optimal vitamin D status: a critical analysis on the basis of evidence-based medicine. J. Clin. Endocrinol. Metab. 98(8), E1283-E1304 (2013)

121. R.J. Heikinheimo, J.A. Inkovaara, E.J. Harju, M.V. Haavisto, R. H. Kaarela, J.M. Kataja, A.M. Kokko, L.A. Kolho, S.A. Rajala, Annual injection of vitamin D and fractures of aged bones. Calcif. Tissue Int. 51(2), 105-110 (1992)

122. D.P. Trivedi, R. Doll, K.T. Khaw, Effect of four monthly oral vitamin $\mathrm{D}_{3}$ (cholecalciferol) supplementation on fractures and mortality in men and women living in the community: randomised double blind controlled trial. BMJ 326(7387), 469 (2003)

123. B. Dawson-Hughes, S.S. Harris, E.A. Krall, G.E. Dallal, Effect of calcium and vitamin D supplementation on bone density in men and women 65 years of age or older. N. Engl. J. Med. 337 (10), 670-676 (1997)
124. E.R. Larsen, L. Mosekilde, A. Foldspang, Vitamin D and calcium supplementation prevents osteoporotic fractures in elderly community dwelling residents: a pragmatic population-based 3-year intervention study. J. Bone Min. Res. 19(3), 370-378 (2004)

125. A. Avenell, J.C. Mak, D. O'Connell, Vitamin D and vitamin D analogues for preventing fractures in post-menopausal women and older men. Cochrane Database Syst. Rev. 2014(4), CD000227 (2014). https://doi.org/10.1002/14651858.CD000227.pub4

126. B.M. Tang, G.D. Eslick, C. Nowson, C. Smith, A. Bensoussan, Use of calcium or calcium in combination with vitamin D supplementation to prevent fractures and bone loss in people aged 50 years and older: a meta-analysis. Lancet 370(9588), 657-666 (2007)

127. R. Jiajue, Y. Jiang, X. Qi, Q. Wang, W. Wang, Y. Pei, X. Wang, W. Huang, X. Zheng, Z. Ning, O. Wang, M. Li, X. Xing, W. Yu, L. Xu, W. Xia, Calciotropic hormones and the prevalence of vertebral fractures in chinese postmenopausal women with vitamin D insufficiency: peking vertebral fracture study. Calcif. Tissue Int. 104, 622-630 (2019). https://doi.org/10.1007/s00223019-00531-2

128. F. Tecilazich, A.M. Formenti, S. Frara, R. Giubbini, A. Giustina, Treatment of hypoparathyroidism. Best. Pract. Res. Clin. Endocrinol. Metab. 32, 955-964 (2018). https://doi.org/10.1016/j. beem.2018.12.002

129. S. Chiavistelli, A. Giustina, G. Mazziotti, Parathyroid hormone pulsatility: physiological and clinical aspects. Bone Res. 3, 14049 (2015). https://doi.org/10.1038/boneres.2014.49

130. S. Bonadonna, A. Burattin, M. Nuzzo, G. Bugari, E.A. Rosei, D. Valle, N. Iori, J.P. Bilezikian, J.D. Veldhuis, A. Giustina, Chronic glucocorticoid treatment alters spontaneous pulsatile parathyroid hormone secretory dynamics in human subjects. Eur. J. Endocrinol. 152, 199-205 (2005). https://doi.org/10.1530/eje. 1.01841

131. S.J. Rubin, J.H. Park, E.N. Pearce, M.F. Holick, D. McAneny, J.P. Noordzij, Vitamin D status as a predictor of postoperative hypocalcemia after thyroidectomy. Otolaryngol. Head Neck Surg. 163, 501-507 (2020). https://doi.org/10.1177/0194599820917907

132. J.L. Hernández, D. Nan, M. Fernandez-Ayala, M. García-Unzueta, M.A. Hernández-Hernández, M. López-Hoyos, P. Muñoz-Cacho, J.M. Olmos, M. Gutiérrez-Cuadra, J.J. Ruiz-Cubillán, J. Crespo, V.M. Martínez-Taboada, Vitamin D status in hospitalized patients with SARS-CoV-2 infection. J. Clin. Endocrinol. Metab. dgaa733 (2020). https://doi.org/10.1210/clinem/dgaa733

133. V.P. Singh, B. Khatua, B. El-Kurdi, C. Rood, Mechanistic basis and therapeutic relevance of hypocalcemia during severe COVID-19 infection. Endocrine 70(3), 461-462 (2020).

134. L. di Filippo, A.M. Formenti, A. Giustina, Hypocalcemia: the quest for the cause of a major biochemical feature of COVID- 19. Endocrine 70, 463-464 (2020). https://doi.org/10.1007/s12020020-02525-9 